\title{
Evaluation of direct methods for power system stability analysis
}

\author{
I. Vokony ${ }^{1}$, Á. Székely ${ }^{2}$, A. Faludi ${ }^{1}$, T. Barbarics ${ }^{2}$ \\ 1. Group of Power Systems and Environment, Dept. of Electric Power Engineering \\ 2. Group of Electromagnetic Theory, Dept. of Broadband Infocommunication and Electromagnetic Theory
}

Faculty of Electrical Engineering, Budapest University of Technology and Economics

1111 Egry József u. 18. Budapest, Hungary

Tel.: +36703379046, +36306409013 ; Fax: +3614633189

e-mail: vokonyistvan@gmail.com; szekely@evtsz.bme.hu

\begin{abstract}
In our paper we briefly summarize the physical concept, calculation method and application possibilities of a selected stability index that is described in detail in the references. We publish our results of examination of the informational value and reliability of the different indices. Method of the examination was a comparison of the index values and critical clearing time values (obtained by timedomain numerical simulation) calculated for several typical fault cases on a small but realistic sample network. Aim of our work is to develop methods for filtering stability calculation cases for network development planning and to provide a possibility of real-time stability monitoring for power system dispatcher use.
\end{abstract}

\section{Key words}

Power systems, simulation, stability analysis, on-line monitoring

\section{Introduction}

Stability assessment of large and complex power systems can be divided into evaluation of static stability, small signal stability and transient stability. In a given operating point, determining the distance from the limit of power transmission capability of the system or calculating the effect of a relatively small disturbance can be performed using load-flow calculations or wellknown, simple methods from linear system theory. Results of these methods are accurate enough, while amount of computation required remains acceptable.

Transient stability calculations have to determine if the machines of the system can reach a new equilibrium after the electromechanical oscillations resulting from a fault or one or more of the machine units will fall out from synchronous operation. For these calculations, a detailed dynamical model is required, on which the simulation of different faults at various locations and several durations can be performed. The number of such cases can be very high, therefore requirements such as suitable accuracy of results, handleable amount of calculation and a format of results easy to evaluate and understand - can often not be met simultaneously. For the detailed, time-domain analysis of transient processes, the available software are based on sophisticated numerical methods for the solution of the network's multiple coupled, nonlinear differential equation systems, which have a relatively simple physical background. Results from such software tools are accurate, but the needed computational capacity can be considerable, and for complex systems, often only a high number of simulations can lead to the correct decision on which fault cases have to be considered at all for stability assessments. Determining the places and types of failures that affect the system mostly requires practice and very good knowledge of the system's behaviour and characteristics, therefore can hardly be automated.

From the above considerations it is clear that measuring or describing „transient stability” of a certain case with one single quantity is by far not a trivial task. For this reason, several „stability indices” have been worked out in the 1980s, common properties of which are following: calculation of the index value for a certain case does not require time-domain simulation, therefore process of calculation is much simpler and faster, but some kind of approximation based on a physical approach is used for dealing with the multi-machine power system model, therefore results are less accurate. Detailed description of these principles can be found in the reference [1], in this paper we only summarize the physical concept and way of calculation for the selected index. For the validation of the results and to obtain information on the limitations of this index we examine the relation of stability index values and critical clearing times for different cases.

According to local practice in Hungary, stability assessment is performed in the phases of long-term network planning, and eventually as part of the daily planning at the TSO. However, Operational Handbook of UCTE prescribes that the TSO should be able to perform calculations that provide results based on the standard stability criteria of UCTE. Parameters of these stability 
calculations shall also be made available to other TSOs for their own model of foreign networks and for performing stability analyses commonly. However, an accepted, applicable and properly parametrized model and the corresponding assessment algorythm for dynamic stability calcualtions is still not available. It is still a question if the commonly used contingency analyses, together with monitoring line loads and voltages give enough information, or the use of stability indices can provide further valuable data on the current extent of dynamic stability of the system.

\section{Approach of Transient Stability Index}

In this section, we will briefly summarize the concept of TSI, that is described in detail in reference [2].

Basic idea is that by calculating the angular accelerations of the machine units at the instant after the examined fault, we will obtain a quantity that somehow characterizes the severeness of disturbance at that node of the network. We can formulate an index from these accelerations that will give information of the disturbance globally on the whole system. Method of calculation is described in the following points:

- Pretend that a $3 \Phi$-fault occurs at the node of $i$-th machine unit at $t=0$. Calculate the angular accelerations of the examined machine units (relative to a reference machine - noted by index $n$ ), and find the maximum of these values:

$$
\varepsilon_{i}^{r}=\max _{k=1 . . n-1}\left|\varepsilon_{k}-\varepsilon_{n}\right|
$$

- Calculate the average angular acceleration of the whole system:

$$
\bar{\varepsilon}=\frac{1}{n} \cdot \sum_{j=1}^{n} \varepsilon_{j}^{r}
$$

- Calculate the difference of each acceleration from this average:

$$
\Delta_{i}=\left|\varepsilon_{i}^{r}-\overline{\mathcal{\varepsilon}}\right|
$$

- After calculating $\Delta_{i}$ for the case of the fault occuring at all examined machines, TSI can be defined as:

$$
T S I=\max _{i=1 . . n} \Delta_{i}
$$

In order to become familiar with the characteristic values, behaviour and meaning of the obtained TSI values, we need to compare the results with time-domain simulation of the same cases. One of the most characteristic results from these simulations is the critical clearing time, i.e. the maximal duration of the fault before clearing, after which all machine units can return to synchronous operation in a new equilibrium.

According to the referred article, we can get reliable information based on the value of the Transient Stability
Index if the correspondance between TSI and CCT values is strictly monotonous, i.e. we can unambiguously estimate critical clearing times.

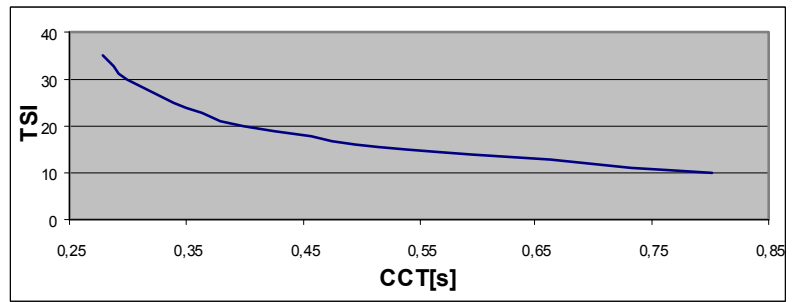

Fig. 1. Theoretically expected dependence of the Transient Stability Index versus critical clearing times.

In our research, we have constructed a reference network for sample calculations in order to check the method and to be able to continue with developing the algorythm that can use output data of standard, on-line load-flow calculations for continuously monitoring TSI values. The next section describes our sample network and details the performed calculations.

\section{The applied sample network model}

We have used a software called Power World Simulator to make the model and perform the calculations. We had to make a model, which is able to simulate almost everything that we can come across in a real power system, i.e. different voltage level buses, transformers, loads, generators, wires, etc. An overview of the sample network is shown Figure 2.

Generators are substituted by an ideal voltage source and a concentrated impedance, parameters of which were calculated using load-flow results from the aboved mentioned software.

$$
E_{g}^{\prime}=U_{s}+\sqrt{3} \cdot i \cdot\left(Z_{g}+Z_{t}\right)
$$

The calculated parameters are summarized in the following table:

\begin{tabular}{|c|c|c|c|c|}
\hline $\operatorname{Re}\{\mathrm{E} g\}[\mathrm{kV}]$ & $\operatorname{Im}\{\mathrm{E} ' \mathrm{~g}\}[\mathrm{kV}]$ & $\operatorname{labs}\left(\mathrm{E}^{\prime} \mathrm{g}\right)[\mathrm{kV}]$ & $\delta \mathrm{g}[\mathrm{rad}]$ & $\delta \mathrm{g}\left[^{\circ}\right]$ \\
\hline 224,366 & 110,938 & 250,294 & 0,459 & 26,323 \\
\hline 118,643 & 54,074 & 130,385 & 0,428 & 24,515 \\
\hline 133,161 & 25,168 & 135,519 & 0,187 & 10,708 \\
\hline 361,923 & 225,740 & 426,552 & 0,558 & 31,969 \\
\hline 406,773 & 0,419 & 406,774 & 0,001 & 0,059 \\
\hline
\end{tabular}

Table 1. Generator parameters of the model network

After we have built up the model, which substitutes the generator, we need a model, which replaces the remaining elements of the in the simplest possible way. It means an impedance and a source. Simplicity of the model makes it more difficult to obtain its parameters. The final model, which we will get for all generators with electric parameters, is depicted on Figure 3. 


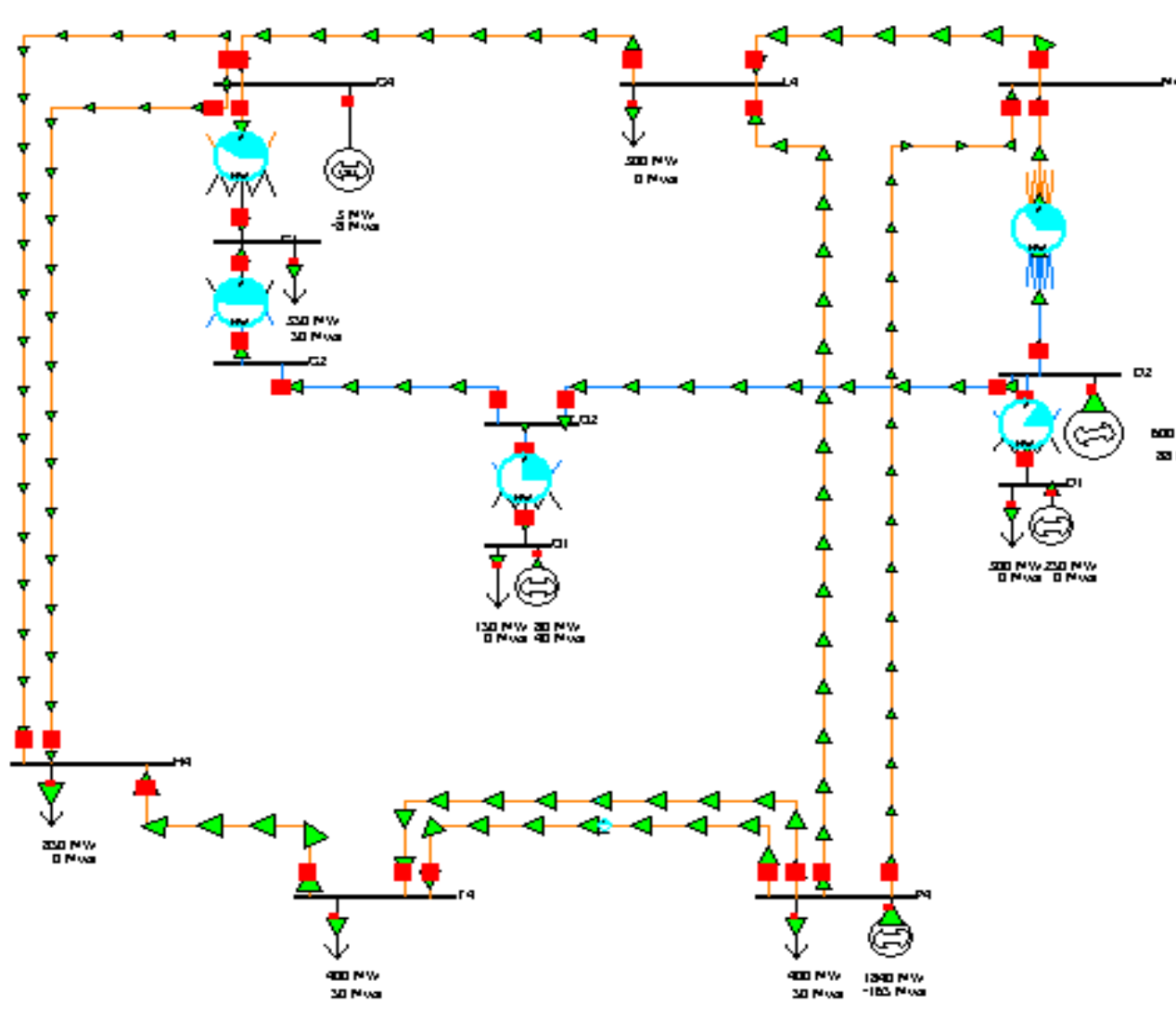

$X_{H}=\frac{U_{n}^{2}}{S_{\text {fault }}}$

This way we calculated all but one of the parameters of our model: voltage and impedance of the generator, impedance of the transformer, and the impedance of the remaining system. The am voltage of the source representing the remaining parts of the system is still to be determined. The impedance of this part is a fixed value. If we use an estate without fault, then the currents on the two sides of Fig. 3., i.e. the ,remaining systems" "current has to be equal to the generators' current. This current induces voltage through the $X_{H}$ impedance. Adding this voltage to the bus voltage will yield the voltage of the source

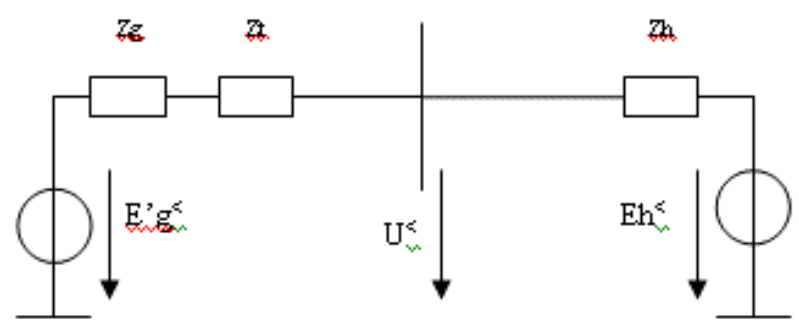

Top of page:

Fig. 2. The model network used for trial calculations

Below:

Fig. 3. The model network used for 3Ф-fault calculations Generator and reduced model of remaining parts of the system.

The first step to get the remaining system-part is a simulation of a $3 \Phi$ - line fault. Such a fault would cause a current flowing on the bus, part of which comes from the generator and the other part from the remaining part of the system. We can calculate the fault current of the generator using our model, load-flow calculations provide the total fault current on the bus, so we can calculate the current which comes from the remaining parts of the system.

The fault current of the generator:

$$
i_{3 \Phi g}=\frac{E_{g}^{\prime}}{\sqrt{3} \cdot\left(Z_{g}+Z_{t}\right)}
$$

If we know the current of the remaining system, we can calculate its power yielded onto the bus, and its impedance:

$$
S_{\text {fault }}=\sqrt{3} \cdot\left|U_{s}\right| \cdot\left|I_{H}^{3 \Phi}\right|
$$

$$
E_{H}=U_{s}-\sqrt{3} \cdot j \cdot X_{H} \cdot i_{g}
$$

\begin{tabular}{|c|c|c|c|}
\hline $\operatorname{Re}\left\{\mathrm{E}^{\prime} h\right\}[\mathrm{kV}]$ & $\operatorname{Im}\left\{\mathrm{E}^{\prime} \mathrm{h}\right\}[\mathrm{kV}]$ & $\operatorname{abs}\left(\mathrm{E}^{\prime} \mathrm{h}\right)[\mathrm{kV}]$ & $\delta \mathrm{h}[\mathrm{rad}]$ \\
\hline 228,759 & $-0,522$ & 228,760 & $-0,002$ \\
\hline 127,549 & 1,420 & 127,557 & 0,011 \\
\hline 121,953 & $-0,951$ & 121,957 & $-0,008$ \\
\hline 470,841 & $-202,790$ & 512,655 & $-0,407$ \\
\hline 408,840 & $-0,287$ & 408,840 & $-0,001$ \\
\hline
\end{tabular}

Table 2. Parameters of the model substituting network parts

The above calculations were implemented in Excel, which is by far not the simplest choice, and changes in input parameters had to be entered manually. In later phases of development of our algorythm we will be able to use this method for checking results.

\section{Results}

We have calculated the index values for different system statuses through the accelerations caused by the faults.

Obtained values are summarized in the following table, where $\Delta \mathrm{t}$ denotes critical clearing time obtained from calculation on our simple model network, CCT denotes that obtained using a time-domain simulation software and TSI is the stability index detailed in section 2. 


\begin{tabular}{|c|c|c|}
\hline$\Delta \mathrm{t}[\mathrm{s}]$ & $\mathrm{CCT}[\mathrm{s}]$ & TSI \\
\hline 0,31 & 0,31 & 18,13 \\
\hline 0,36 & 0,32 & 18,92 \\
\hline 0,44 & 0,32 & 19,83 \\
\hline 0,55 & 0,32 & 20,63 \\
\hline 0,73 & 0,33 & 21,42 \\
\hline 1,12 & 0,33 & 22,20 \\
\hline 0,31 & 0,36 & 18,82 \\
\hline 0,39 & 0,46 & 12,98 \\
\hline 0,52 & 0,61 & 7,12 \\
\hline 0,80 & 0,95 & 4,84 \\
\hline 0,63 & 0,67 & 4,29 \\
\hline 0,81 & 0,80 & 2,57 \\
\hline 1,21 & 1,25 & 3,49 \\
\hline 0,25 & 0,33 & 6,33 \\
\hline 0,30 & 0,37 & 4,83 \\
\hline 0,37 & 0,42 & 5,66 \\
\hline 0,46 & 0,53 & 6,52 \\
\hline 0,60 & 0,66 & 7,36 \\
\hline 0,82 & 0,71 & 8,23 \\
\hline 1,29 & 0,72 & 9,04 \\
\hline & & \\
\hline
\end{tabular}

Table 3. Transient Stability Index values compared with critical clearing times for different fault cases

The most representative lines are marked with yellow. We have made some neglections, e.g. we monitored the instability only at the generator where the fault occurs. However, in some of the cases, we get the largest angular acceleration on a generator other than that. Next step in our research will be to handle these cases without directly applying time-domain simulation results.

The results of the ,realistic" cases are plotted on the following figure:

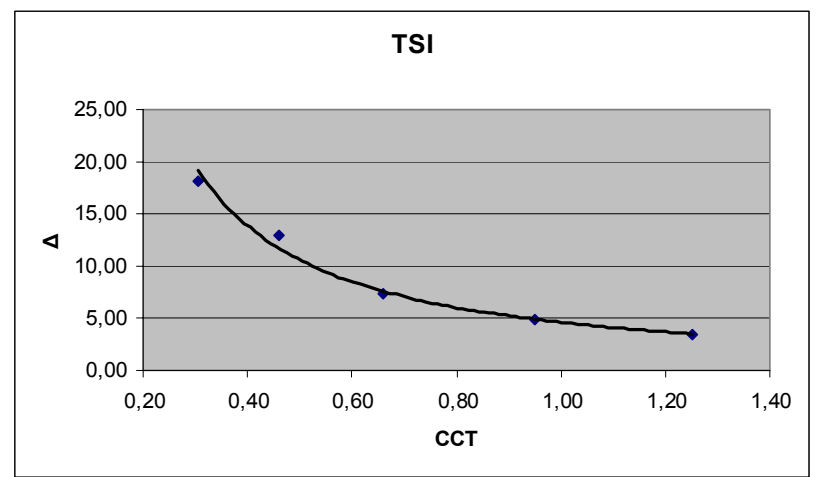

Fig. 4. Dependence of the Transient Stability Index versus critical clearing times - results from calculation.

From the results, it is clear that for some of the cases, TSI provided relevant and valuable information on the dynamic security of the system, while in other cases, neglections in our oversimplified model have yielded unrealistic results. We are going to continue working on the solution of these problems.

\section{Conclusion}

- The charasteristic shows a clearly monotonous dependence between the transient stability index values and the critical clearing time.

- It's possible to give information on dynamic security of particular system states based on the value of the examined index.

- The index was originally created to reduce the required amount of calculations by (partially) substituting complicated time.domain analyses. Nowadays this aim has lost its importance because of the growth of available computational capacity. However, stability indices can be very useful to monitor stability state of the system on-line by automatically calculating an easily interpretable index value instead of evaluating hundreds of time-fuctions obtained from simulation.

Our future research plans on this topic include:

- Developing a less complicated algorythm for calculating the accelerations for the generators other than those closest to the examined fault.

- Applying network analysis methods for automated calculation of necessary network parameters

- Merging the two sub-algorythms into one that directly calculates stability index values using load-flow results as an input

- Analysing other stability indices

- Examining the possibility of usage of such indices for TSOs, dispatchers, etc.

\section{Acknowledgement}

The authors wish to thank for the financial support provided for this research project by the Foundation for the Education of Energy Engineers.

\section{References}

[1] A.A. Fouad, K.C. Kruempel, V. Vittal, A. Ghafurian, K. Nodehi, J. V. Mitsche:

Transient Stability Program Output Analysis.

IEEE Trans. on Power Systems, Vol. PWRS-1, No.1, 1986.

[2] M. Ribbens-Pavella, P. G. Murthy, J. L. Horward, J. L. Carpentier:

Transient Stability Index for on-line stability assessment and contingency evaluation. Intl. Journal of Power and Energy Systems, Vol. 4. № 2, Apr. 1982.

[3] L. Szabó - A. Faludi: Operation and control of power systems. University textbook. BME, Budapest, 2002. 